\title{
Vibrations in binary and ternary topological insulators: First-principles calculations and Raman spectroscopy measurements
}

\author{
V. Chis \\ Donostia International Physics Center (DIPC), P. Manuel de Lardizabal 4, Donostia-San Sebastián ES-20018, Basque Country, Spain
}

I. Yu. Sklyadneva

Donostia International Physics Center (DIPC), P. Manuel de Lardizabal 4, Donostia-San Sebastián ES-20018, Basque Country, Spain, Institute of Strength Physics and Material Science, pr. Academicheskii, 2/1, 634021 Tomsk, Russian Federation, and Tomsk State University, 6340501 Tomsk, Russian Federation

K. A. Kokh

Institute of Geology and Mineralogy, SB RAS, Novosibirsk 630090, Russian Federation

\author{
V. A. Volodin and O. E. Tereshchenko \\ Institute of Semiconductor Physics, Novosibirsk 630090, Russian Federation and \\ Novosibirsk State University, Novosibirsk 636090, Russian Federation
}

E. V. Chulkov

Donostia International Physics Center (DIPC), P. Manuel de Lardizabal 4, Donostia-San Sebastián ES-20018, Basque Country, Spain, Departemento de Fisíca de Materiales, Facultad de Ciencias Químicas, UPV/EHU, Apdo. 1072, Donostia-San Sebastián ES-20080, Basque Country, Spain, and

Centro de Fisíca de Materiales, CFM-Materials Physics Center MPC, Centro Mixto CSIC-UPV/EHU,

Donostia-San Sebastián ES-20018, Basque Country, Spain

(Received 22 June 2012; revised manuscript received 22 October 2012; published 19 November 2012)

\begin{abstract}
Density-functional perturbation theory and Raman spectroscopy are used in the study of vibrations in bulk $\mathrm{V}_{2} \mathrm{VI}_{3}$ binary and ternary topological insulators as well as in ultrathin films composed of one and two quintuple layers. Full dispersion curves along the Brillouin zone symmetry directions are calculated and analyzed and the zonecenter Raman active modes are compared to the experimental results. The calculations of the two quintuple layer films reveal modes that correspond to displacements of entire quintuple layer blocks. These modes have low frequencies and are Raman active. The phonon dispersion curves of one and two quintuple layer $\mathrm{Bi}_{2} \mathrm{Se}_{3}$ films are compared with recent helium atom scattering experiments [Phys. Rev. Lett. 107, 186102 (2011)].
\end{abstract}

DOI: 10.1103/PhysRevB.86.174304

PACS number(s): 63.20.D-, 78.30.-j, 68.35.Ja

\section{INTRODUCTION}

The binary compounds $\mathrm{Bi}_{2} \mathrm{Se}_{3}, \mathrm{Bi}_{2} \mathrm{Te}_{3}$, and $\mathrm{Sb}_{2} \mathrm{Te}_{3}$, and ternary systems $\mathrm{Bi}_{2} \mathrm{Se}_{2} \mathrm{Te}, \mathrm{Bi}_{2} \mathrm{Se}_{2} \mathrm{~S}, \mathrm{Bi}_{2} \mathrm{Te}_{2} \mathrm{Se}, \mathrm{Bi}_{2} \mathrm{Te}_{2} \mathrm{~S}$, $\mathrm{Sb}_{2} \mathrm{Te}_{2} \mathrm{Se}$, and $\mathrm{Sb}_{2} \mathrm{Te}_{3} \mathrm{~S}$ are materials with a large range of potential applications. Most of them are classified as topological insulators that exhibit an insulating narrow gap in the bulk. When the three-dimensional (3D) symmetry is broken these materials behave as metals in the surface region characterized by a single electronic surface state that has a Dirac-like dispersion around the surface Brillouin zone center ( $\bar{\Gamma}$ point). This surface state is topologically protected against back scattering and arises due to strong spin-orbit coupling. The electronic properties of the binary compounds have been thoroughly investigated over the recent years both experimentally ${ }^{1-4}$ and theoretically, ${ }^{5-10}$ while the ternary compounds have been studied more sparsely. ${ }^{11-14}$ The composition of the ternary compounds we study is characterized by a substitution of one atom in the binary compound by a different atom such as Se, Te, or S. As of this moment only the composition of $\mathrm{Bi}_{2} \mathrm{Se}_{2} \mathrm{~S}^{13,15}$ has failed to show any character of topologically protected surface state.

The large interest in the topological materials resides not only in fundamental research but also in the promising properties of these materials for future applications. Possible applications have been mentioned to be within spintronics, quantum computing, magnetic memories, and thermoelectric materials. ${ }^{16,17}$ The binary compounds $\mathrm{Bi}_{2} \mathrm{Se}_{3}, \mathrm{Bi}_{2} \mathrm{Te}_{3}$, and $\mathrm{Sb}_{2} \mathrm{Te}_{3}$ have proved to be excellent thermoelectric materials and the thermoelectric performance has been attributed to the details of the narrow gap electronic structure combined with low lattice thermal conductivity. It remains unclear if the ternary compounds would perform as well as the binary compounds since the thermoelectric properties of these compounds have not been measured or reported. For optimal thermoelectric figure of merit $(Z T)$, materials require a large thermopower, high electric conductivity, and low thermal conductivity. With this purpose in mind thin films are most suitable and widely used materials in thermoelectric devices. Growth of thin films is done by several techniques such as the vapor-liquid-solid process, ${ }^{18}$ molecular beam epitaxy, ${ }^{19}$ and mechanical exfoliation ${ }^{20,21}$ of thin sheets from bulk crystals and vapor-phase deposition by van der Waals epitaxy. ${ }^{22}$

Vibrational properties of bulk and thin film compounds are widely studied by Raman spectroscopy. For $\mathrm{Bi}_{2} \mathrm{Se}_{3}, \mathrm{Bi}_{2} \mathrm{Te}_{3}$, and $\mathrm{Sb}_{2} \mathrm{Te}_{3}$ Raman $^{20-25}$ spectroscopy measurements have been performed ranging from a few quintuple layers to bulk size crystals. Besides Raman spectroscopy measurements inelastic 
neutron scattering ${ }^{26}$ experiments were performed on powder samples of $\mathrm{Bi}_{2} \mathrm{Se}_{3}$ and $\mathrm{Bi}_{2} \mathrm{Te}_{3}$ and a recently published study of surface phonon modes within the surface Brillouin zone of the $\mathrm{Bi}_{2} \mathrm{Se}_{3}$ surface was carried out in terms of helium atom scattering ${ }^{27}$ (HAS). The vibrational properties of binary compounds, $\mathrm{Bi}_{2} \mathrm{Se}_{3},{ }^{28,29} \mathrm{Bi}_{2} \mathrm{Te}_{3},{ }^{28-30}$ and $\mathrm{Sb}_{2} \mathrm{Te}_{3}{ }^{31}$ has previously been evaluated for both bulk and/or thin film structures. However, there are no studies of the vibrational properties based on first-principles calculations for binary compounds except for bulk $\mathrm{Sb}_{2} \mathrm{Te}_{3} .{ }^{31}$ In addition, we are not aware of any study of vibrational properties of the ternary compounds.

This paper is organized in the following. In Secs. II and III we address the technical aspects regarding sample growth of binary and ternary compounds, the setup for Raman spectroscopy and the calculation details of vibrational properties. In Sec. IV we show and analyze our experimental and computational results. The main focus will be on the zone-center Raman active phonon modes for the bulk binary and ternary compounds. In the second part of Sec. IV we present the calculation results of vibrational properties for binary and ternary thin films, while the last part of this section will include comparison of the surface phonon modes of $\mathrm{Bi}_{2} \mathrm{Se}_{3}$ with recent HAS experiments. Finally, in Sec. V the conclusions are drawn.

\section{EXPERIMENTAL DETAILS}

Single crystals of $\mathrm{V}_{2} \mathrm{VI}_{3-x} \mathrm{VI}_{x}(\mathrm{~V}=\mathrm{Bi}, \mathrm{Sb} ; \mathrm{VI}=\mathrm{Se}, \mathrm{Te}, \mathrm{S})$ were prepared from elementary $\mathrm{Bi}, \mathrm{Sb}, \mathrm{Te}, \mathrm{Se}$, and $\mathrm{S}$ of $99.999 \%$ purity. The synthesis of the binary $\mathrm{Bi}_{2} \mathrm{Te}_{3}, \mathrm{Bi}_{2} \mathrm{Se}_{3}$, and $\mathrm{Sb}_{2} \mathrm{Te}_{3}$ as well as ternary $\mathrm{Bi}_{2} \mathrm{Te}_{2} \mathrm{~S}, \mathrm{Sb}_{2} \mathrm{Te}_{2} \mathrm{Se}, \mathrm{Sb}_{2} \mathrm{Te}_{2} \mathrm{~S}$ compounds was done by direct alloying of stoichiometric charges in the sealed quartz ampoules by heating at a rate of $20^{\circ} \mathrm{C} / \mathrm{h}$ until the temperature reached $20^{\circ} \mathrm{C}$ above the melting point. The melting points of $\mathrm{Bi}_{2} \mathrm{Te}_{3}, \mathrm{Bi}_{2} \mathrm{Se}_{3}, \mathrm{Sb}_{2} \mathrm{Te}_{3}$, $\mathrm{Bi}_{2} \mathrm{Te}_{2} \mathrm{~S}, \mathrm{Sb}_{2} \mathrm{Te}_{2} \mathrm{Se}$, and $\mathrm{Sb}_{2} \mathrm{Te}_{2} \mathrm{~S}$ materials are 585, 705, 620, 630,630 , and $650{ }^{\circ} \mathrm{C}$, respectively. Presynthesized compounds and mixtures of $\mathrm{Bi}_{2} \mathrm{Se}_{3}$ and $\mathrm{Bi}_{2} \mathrm{Te}_{3}$ were put in carbon-coated single-wall 7-mm-diameter ampoules with conical tip and were sealed under $10^{-4}$ torr. Starting compositions were chosen with a $\mathrm{Bi}_{2} \mathrm{Te}_{3}: \mathrm{Bi}_{2} \mathrm{Se}_{3}$ ratio of $2: 1\left(\mathrm{Bi}_{2} \mathrm{Te}_{2} \mathrm{Se}\right)$ and 1:2 $\left(\mathrm{Bi}_{2} \mathrm{Se}_{2} \mathrm{Te}\right)$, respectively. For recrystallization we used vertical variant of the modified Bridgman method with rotating heat field. ${ }^{32}$ Prior to pulling the ampoules were heated to $70{ }^{\circ} \mathrm{C}$ above the melting point for a half a day homogenization. Melting temperatures for $\mathrm{Bi}_{2} \mathrm{Se}_{2} \mathrm{Te}$ and $\mathrm{Bi}_{2} \mathrm{Te}_{2} \mathrm{Se}$ were considered to be $665^{\circ} \mathrm{C}$ and $625^{\circ} \mathrm{C}$, correspondingly. After pulling the ampoules through the vertical temperature gradient $\sim 15^{\circ} \mathrm{C} / \mathrm{cm}$ with a rate of $10 \mathrm{~mm} /$ day the furnace was switched off. The resulted ingots $10 \mathrm{~cm}$ in length consisted of one or several large single crystalline blocks.

Raman spectra were measured in a Jobin Yvon T64000 spectrometer (micro-Raman, in the $20-1000 \mathrm{~cm}^{-1}$ Raman shift range, with a spot size of $\sim 30 \mu \mathrm{m}$ and spectral resolution of about $\sim 1.5 \mathrm{~cm}^{-1}$ ). In all cases, Raman spectra were recorded at room temperature in a backscattering geometry using a 514.5-nm line of an $\mathrm{Ar}^{+}$laser with polarized incident light and with analyzer of scattered light. "Parallel" polarization geometry (the polarization of scattered light was parallel to the polarization of incident light (VV)) and "perpendicular" polarization geometry [the polarization of scattered light was perpendicular to the polarization of incident light (UV)] were used. All spectra were recorded at low power levels $P<3 \mathrm{~mW}$ in order to avoid local heating by the laser spot. The active Raman modes in $\mathrm{V}_{2} \mathrm{VI}_{3}$ materials are observed with frequencies in the $20-200 \mathrm{~cm}^{-1}$ range in which peaks associated with the rotational and vibrational modes of the nitrogen and oxygen molecules in air are present. These additional peaks originating from the airborne nitrogen and oxygen molecules were subtracted from the resulting spectra of the $\mathrm{V}_{2} \mathrm{VI}_{3}$ materials by recording the spectra from bulk silicon in the corresponding geometry (VV and UV). The silicon wafer is suitable as a reference since there are no Raman peaks detected in the $20-200 \mathrm{~cm}^{-1}$ range.

\section{COMPUTATIONAL DETAILS}

The first-principles calculations were performed with the total energy, plane-wave code within the QUANTUMESPRESSO $^{33}$ (QE) package which is based on the densityfunctional theory (DFT) and density-functional perturbation theory (DFPT). We adopt scalar relativistic ultrasoft pseudopotentials generated with the Rappe-Rabe-Kaxiras-Joannopoulos recipe $^{34,35}$ and either the local density approximation ${ }^{36}$ (LDA) or the general gradient approximation ${ }^{37}$ (GGA) for the exchange and correlation energy. The electronic wave functions were expanded in plane waves with an energy cutoff of $35 \mathrm{Ry}$ while for the effective potential and the charge density the energy cutoff has been taken equal to $500 \mathrm{Ry}$. The integration over the Brillouin zone is carried out over a $(9 \times 9 \times 9)$ and a $(12 \times 12 \times 1) \mathbf{k}$-point grid of the Monkhorst-Pack division for the bulk structures and for the thin films, respectively. All calculations were performed for fully optimized internal structure and lattice parameters.

The family class of $\mathrm{V}_{2} \mathrm{VI}_{3}$ materials form a rhombohedral layered lattice (see Fig. 1) in which the unit cell contains building blocks of quintuple layers (QLs) in the following sequence,

$$
\ldots-[\mathrm{VI}]-[\mathrm{V}]-[\mathrm{VI}]-[\mathrm{V}]-[\mathrm{VI}]-[\mathrm{VI}]^{\prime}-\ldots,
$$

where $[\mathrm{VI}]^{\prime}$ is the beginning of the adjacent QL. One unit cell with rhombohedral crystal symmetry $\left(D_{3 d}^{5}\right.$ symmetry and space group R $\overline{3} \mathrm{~m}$ ) comprises three QLs. The optimized bulk lattice parameters and the internal structures are summarized in Table I together with available experimental values. In most of the cases the optimized lattice parameters are very close to the experimental values, within $1 \%-2 \%$. The quintuple layer (QL) thin films were constructed from the optimized bulk terminated structure. One and two QLs are constructed by slabs including vacuum separating adjacent surfaces. The vacuum intervals are set to $16 \AA$ along the $z$ direction. The atomic structure of bulk materials and QLs were optimized until the forces acting on each atom were less then $0.25 \mathrm{meV} / \AA$. The dynamical properties of the bulk structures and the QLs were calculated by evaluating the dynamical matrices over a $(4 \times 4 \times 4)$ and a $(6 \times 6 \times 1)$ mesh of $\mathbf{q}$ point, respectively.

The calculation of phonon dispersion curves is carried out without the inclusion of the spin-orbit coupling. For several other properties of the topological insulator materials 


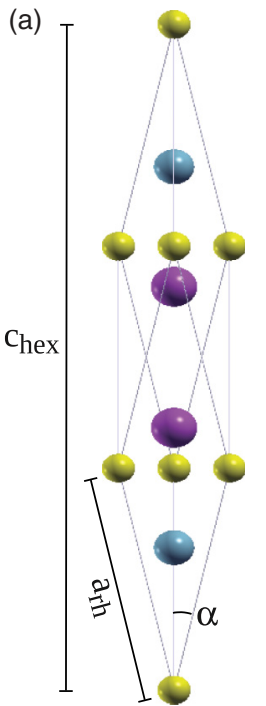

(b)

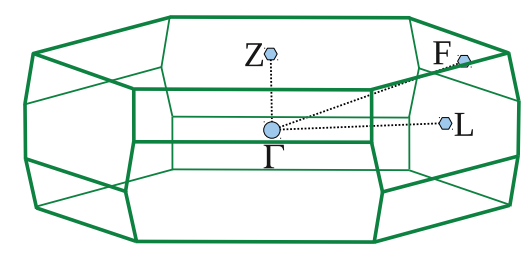

Selenium

Tellurium

Bismuth
FIG. 1. (Color online) Rhombohedral unit cell for $\mathrm{V}_{2} \mathrm{VI}_{3}$ family class compounds illustrated by $\mathrm{Bi}_{2} \mathrm{Te}_{2} \mathrm{Se}(\mathrm{a})$, and the corresponding Brillouin zone with the high symmetry points and directions outlined in (b). The internal structure and lattice parameters of the rhombohedral structure are listed in Table I.

the spin-orbit interaction is crucial, for example, electronic properties. However, as it turns out the spin-orbit interaction is less important in describing the vibrational properties of the compounds. We have carried out tests where the phonon dispersion curves have been calculated with and without the inclusion of spin-orbit interaction with the result of only small changes in the phonon frequencies yet the characters of the phonon branches are preserved. In this paper the results of one $\mathrm{QL}$ of $\mathrm{Bi}_{2} \mathrm{Se}_{3}$ including SO interaction will be addressed.

\section{RESULTS}

\section{Dynamical properties of bulk structures}

The Raman spectra of the $\mathrm{V}_{2} \mathrm{VI}_{3}$ materials recorded in the two polarized scattering geometries and fixed power levels are shown in Figs. 2 and 3 and the phonon dispersion curves calculated along the main symmetry directions are presented in Fig. 4. Both the calculated and experimental Raman active modes in the $\Gamma$ point are summarized in Table II.
Phonons at the $\Gamma$ point are classified according to the irreducible representation of the point group $D_{3 d}^{5}$ as

$$
\Gamma=2\left(A_{1 g}+E_{g}\right)+3\left(E_{u}+A_{2 u}\right) .
$$

Two $E_{u}$ and one $A_{2 u}$ are acoustic modes. The displacement patterns of the corresponding $\Gamma$-point modes are shown in Fig. 5 in their primitive cell representation. The modes which display a dipole moment ( $\mathrm{u}$ modes) couple to the inner macroscopic longitudinal electric field which shifts the LO phonon frequencies. The macroscopic field contribution to the dynamical matrix introduces an angular dispersion of the phonons at the $\Gamma$ point, that is, the limit of the phonon bands $\omega(q)$ for $q \rightarrow 0$ depends on the angle formed by $q$ with the optical axis. According to the classification of the $\Gamma$-point modes, 15 modes in total, three are acoustic and 12 are optical modes, respectively. Four of the 12 optical modes are Raman active, $2 E_{g}+2 A_{1} g$, with frequencies in the $30-200 \mathrm{~cm}^{-1}$ range.

Figures 2 and 3 show that the Raman spectra obtained from bulk crystals exhibit mainly three Raman active modes in the ranges of $60-80,100-135$, and $135-180 \mathrm{~cm}^{-1}$, which can be identified as related to symmetry $A_{1 g}^{1}, E_{g}^{2}$, and $A_{1 g}^{2}$, respectively. The peak positions for $\mathrm{Bi}_{2} \mathrm{Te}_{3}, \mathrm{Bi}_{2} \mathrm{Se}_{3}$, and $\mathrm{Sb}_{2} \mathrm{Te}_{3}$ are very close to the previously reported characteristic Raman vibration mode positions for bulk crystalline materials. ${ }^{39-41}$ On some samples we observed a very weak line at $35-45 \mathrm{~cm}^{-1}$, corresponding to the $E_{1 g}$ mode, ${ }^{42}$ which was difficult to separate from the overlapping Raman peaks from air. $\mathrm{In}_{2} \mathrm{Te}_{3}$, low frequency $A_{1 g}^{1}$ and high frequency $E_{g}^{2}$ and $A_{1 g}^{2}$ modes are detected at $61.5,101.5$, and $133.5 \mathrm{~cm}^{-1}$, respectively. $\mathrm{In}_{\mathrm{Bi}_{2}} \mathrm{Se}_{3}$, the same modes are detected at 72.5, 131.5 , and $176.5 \mathrm{~cm}^{-1}$, respectively. Figure 2 shows that in the case of Te replacement by Se or S, the phonon peaks exhibit blue shift as expected, but the value of the shift is different for the three modes. The low and high frequency $A_{1 g}$ modes have a distinct behavior and value of the shift with the change of composition. Note that for the Sb-based ternary crystals an agreement with theory is worse than for the Bi-based materials and can be explained by the more defected structure of the bulk Sb-based ternary compounds.

In Fig. 4 the phonon dispersion curves are characterized by three acoustic modes that in most of the cases involve mainly $\mathrm{Bi} / \mathrm{Sb}$ atoms in their displacements. In the direction $Z-\Gamma$ the three low energy phonon modes correspond to two degenerate $E_{u}$ modes and one $A_{2 u}$ mode. The frequencies of these modes

TABLE I. Optimized lattice parameters and internal structures for the rhombohedral crystal structure compared to the available experimental data.

\begin{tabular}{lccccccccc}
\hline \hline & $\mathrm{Bi}_{2} \mathrm{Se}_{3}$ & $\mathrm{Bi}_{2} \mathrm{Te}_{3}$ & $\mathrm{Bi}_{2} \mathrm{Se}_{2} \mathrm{~S}$ & $\mathrm{Bi}_{2} \mathrm{Se}_{2} \mathrm{Te}$ & $\mathrm{Bi}_{2} \mathrm{Te}_{2} \mathrm{~S}$ & $\mathrm{Bi}_{2} \mathrm{Te}_{2} \mathrm{Se}$ & $\mathrm{Sb}_{2} \mathrm{Te}_{3}$ & $\mathrm{Sb}_{2} \mathrm{Te}_{2} \mathrm{Se}$ & $\mathrm{Sb}_{2} \mathrm{Te}_{2} \mathrm{~S}$ \\
\hline $\mathrm{a}_{\text {hex }}(\AA)$ & 4.10 & 4.40 & 4.14 & 4.21 & 4.23 & 4.31 & 4.22 & 4.14 & 4.09 \\
$\mathrm{a}_{\text {hex }}(\AA)^{38}$ & 4.14 & 4.38 & - & 4.22 & 4.32 & 4.28 & 4.25 & 4.20 & - \\
$\mathrm{c}_{\text {hex }}(\AA)$ & 28.33 & 29.71 & 29.75 & 29.20 & 29.60 & 29.9 & 30.12 & 29.54 & 29.18 \\
$\mathrm{c}_{h e x}(\AA)^{38}$ & 28.64 & 30.50 & - & 29.24 & 30.01 & 29.86 & 30.35 & 29.94 & - \\
$\mathrm{a}_{r h}(\AA)$ & 9.74 & 10.22 & 9.83 & 10.03 & 10.16 & 10.27 & 10.33 & 10.133 & 10.33 \\
$\mathrm{a}_{r h}(\AA)^{38}$ & 9.84 & 10.47 & - & 10.05 & 10.31 & 10.26 & 10.43 & 10.27 & - \\
$\alpha$ & $24.31^{\circ}$ & $24.80^{\circ}$ & $24.33^{\circ}$ & $24.22^{\circ}$ & $24.04^{\circ}$ & $24.19^{\circ}$ & $23.58^{\circ}$ & $23.57^{\circ}$ & $23.59^{\circ}$ \\
$\alpha^{38}$ & $24.27^{\circ}$ & $24.17^{\circ}$ & - & $24.24^{\circ}$ & $24.17^{\circ}$ & $24.09^{\circ}$ & $23.52^{\circ}$ & $23.53^{\circ}$ & - \\
\hline \hline
\end{tabular}




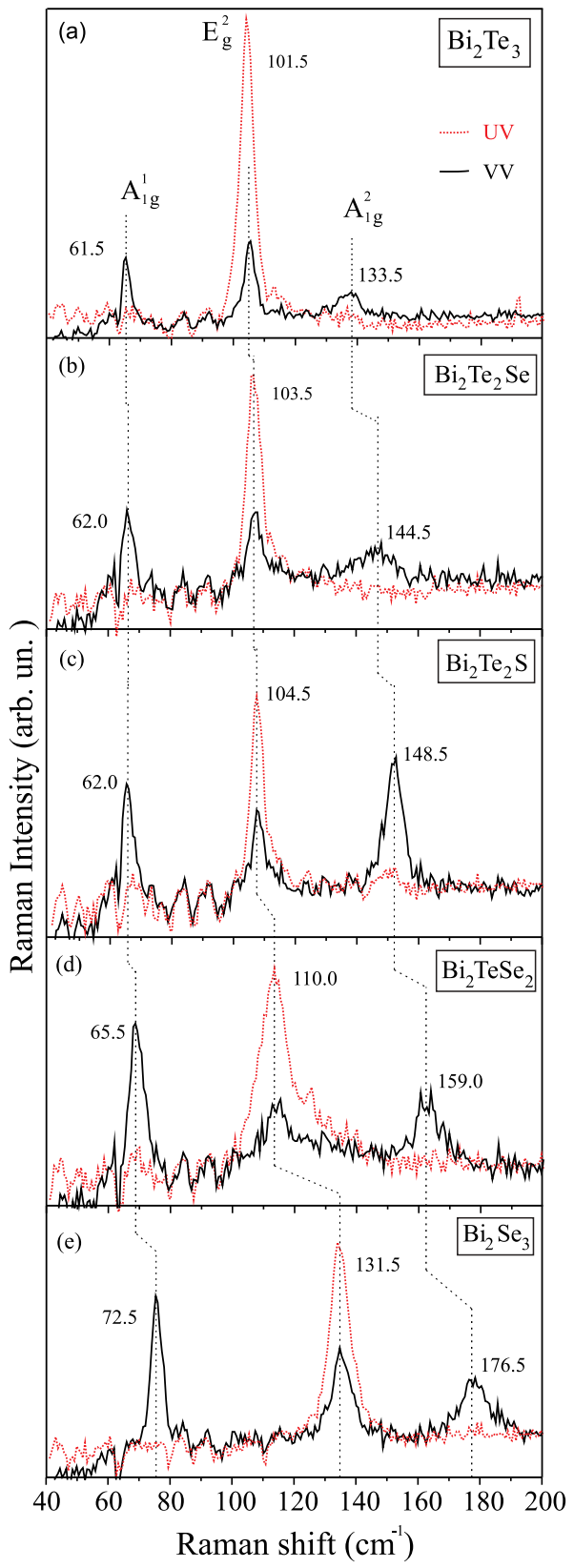

FIG. 2. (Color online) Raman spectra of the bismuth based $\mathrm{V}_{2} \mathrm{VI}_{3}$ bulk crystals: (a) $\mathrm{Bi}_{2} \mathrm{Te}_{3}$, (b) $\mathrm{Bi}_{2} \mathrm{Te}_{2} \mathrm{Se}$, (c) $\mathrm{Bi}_{2} \mathrm{Te}_{2} \mathrm{~S}$, (d) $\mathrm{Bi}_{2} \mathrm{Se}_{2} \mathrm{Te}$, and (e) $\mathrm{Bi}_{2} \mathrm{Se}_{3}$. Spectra measured in the geometry when the polarization of scattered light was parallel (VV, black solid line) and perpendicular (UV, red dotted line) to the polarization of the incident light are shown.

at the $Z$ point vary with respect to different atomic species present in the compound.

The Raman active $E_{g}^{1}$ mode at the $\Gamma$ point [Fig. 5(a)] for the binary compounds is found at frequencies according to Table II where for $\mathrm{Bi}_{2} \mathrm{Te}_{3}$ the $E_{g}^{1}$ frequency is found to be the lowest among the studied compounds and $\mathrm{Sb}_{2} \mathrm{Te}_{3}$ experiences the highest $E_{g}^{1}$ mode frequency. For a large $\mathrm{Bi}_{2} \mathrm{Te}_{3}$ nanoparticle ${ }^{23}$ the $E_{g}^{1}$ mode was reported at $37 \mathrm{~cm}^{-1}$, in good agreement with our calculations, while in the second report for $\mathrm{Bi}_{2} \mathrm{Te}_{3}{ }^{24}$ the $E_{g}^{1}$ mode is found at $34.4 \mathrm{~cm}^{-1}$, which is slightly lower than the calculated one. The order of the frequencies for the

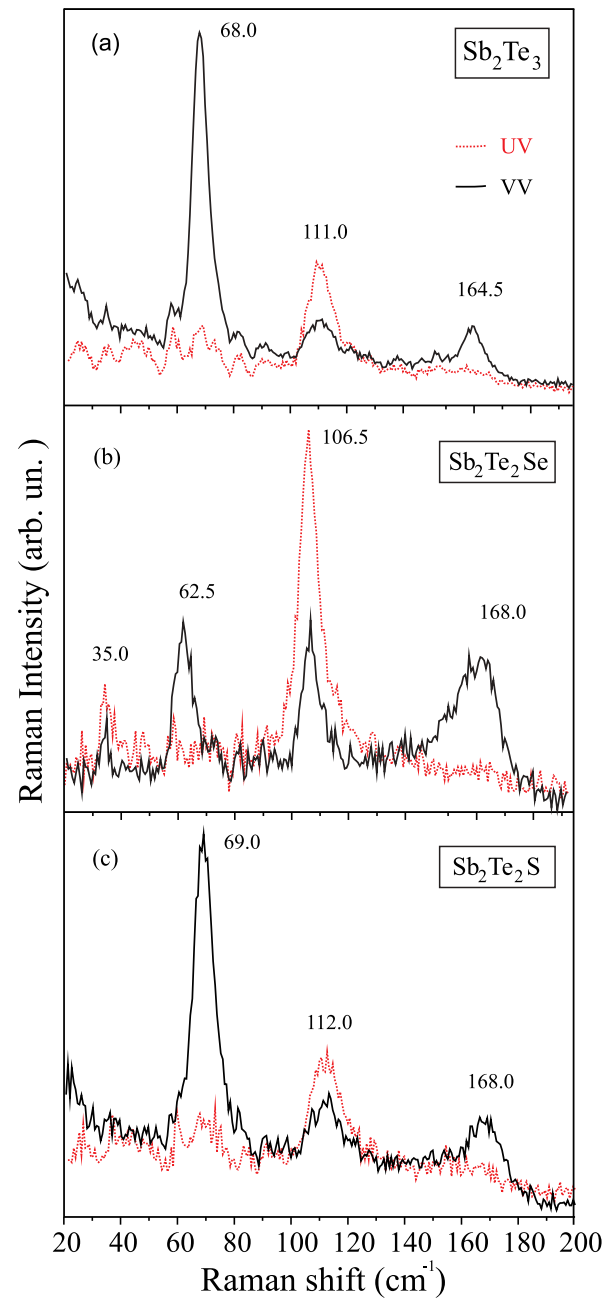

FIG. 3. (Color online) Raman spectra of the antimony based $\mathrm{V}_{2} \mathrm{VI}_{3}$ bulk crystals: (a) $\mathrm{Sb}_{2} \mathrm{Te}_{3}$, (b) $\mathrm{Sb}_{2} \mathrm{Te}_{2} \mathrm{Se}$, (c) $\mathrm{Sb}_{2} \mathrm{Te}_{2} \mathrm{~S}$. Spectra measured in the geometry when the polarization of scattered light was parallel (VV, black solid line) and perpendicular (UV, red dotted line) to the polarization of the incident light are shown.

binary compounds follows the atomic mass of the participating atoms in the $E_{g}^{1}$ mode displacement field [Fig. 5(a)], where Bi is the heaviest atom followed by $\mathrm{Te}, \mathrm{Sb}$, and finally $\mathrm{Se}$. The displacement field of the $E_{g}^{1}$ mode involves the two topmost atomic layers within the QL building blocks while the middle atomic layer is at rest. For the ternary compounds, it is found that $\mathrm{Bi}_{2} \mathrm{Te}_{2} \mathrm{Se}$ experiences the lowest $E_{g}^{1}$ frequency followed by $\mathrm{Bi}_{2} \mathrm{Te}_{2} \mathrm{~S}, \mathrm{Bi}_{2} \mathrm{Se}_{2} \mathrm{Te}, \mathrm{Bi}_{2} \mathrm{Se}_{2} \mathrm{~S}, \mathrm{Sb}_{2} \mathrm{Te}_{2} \mathrm{~S}$, and $\mathrm{Sb}_{2} \mathrm{Te}_{2} \mathrm{Se}$ showing the highest $E_{g}^{1}$ frequency among the compounds. The explanation for the binary compounds can be applied as well for the ternary compounds although the situation is more complicated since the surrounding environment for the ternary compounds of the participating atoms must be taken into account. As an example, the difference in frequency between $\mathrm{Bi}_{2} \mathrm{Te}_{2} \mathrm{Se}$ and $\mathrm{Bi}_{2} \mathrm{Te}_{2} \mathrm{~S}$ is explained by the different bond length between the Bi layer and the middle layer ( $\mathrm{Se}$ or $\mathrm{S}$ ). For $\mathrm{Bi}_{2} \mathrm{Te}_{2} \mathrm{~S}$ the bond is somewhat shorter, which results in a stiffening of the corresponding force constant.

The dispersion of the $E_{g}^{1}$ mode throughout the $\mathrm{BZ}$ is very flat for almost all compounds in this study. For $\mathrm{Bi}_{2} \mathrm{Se}_{3}$ the 

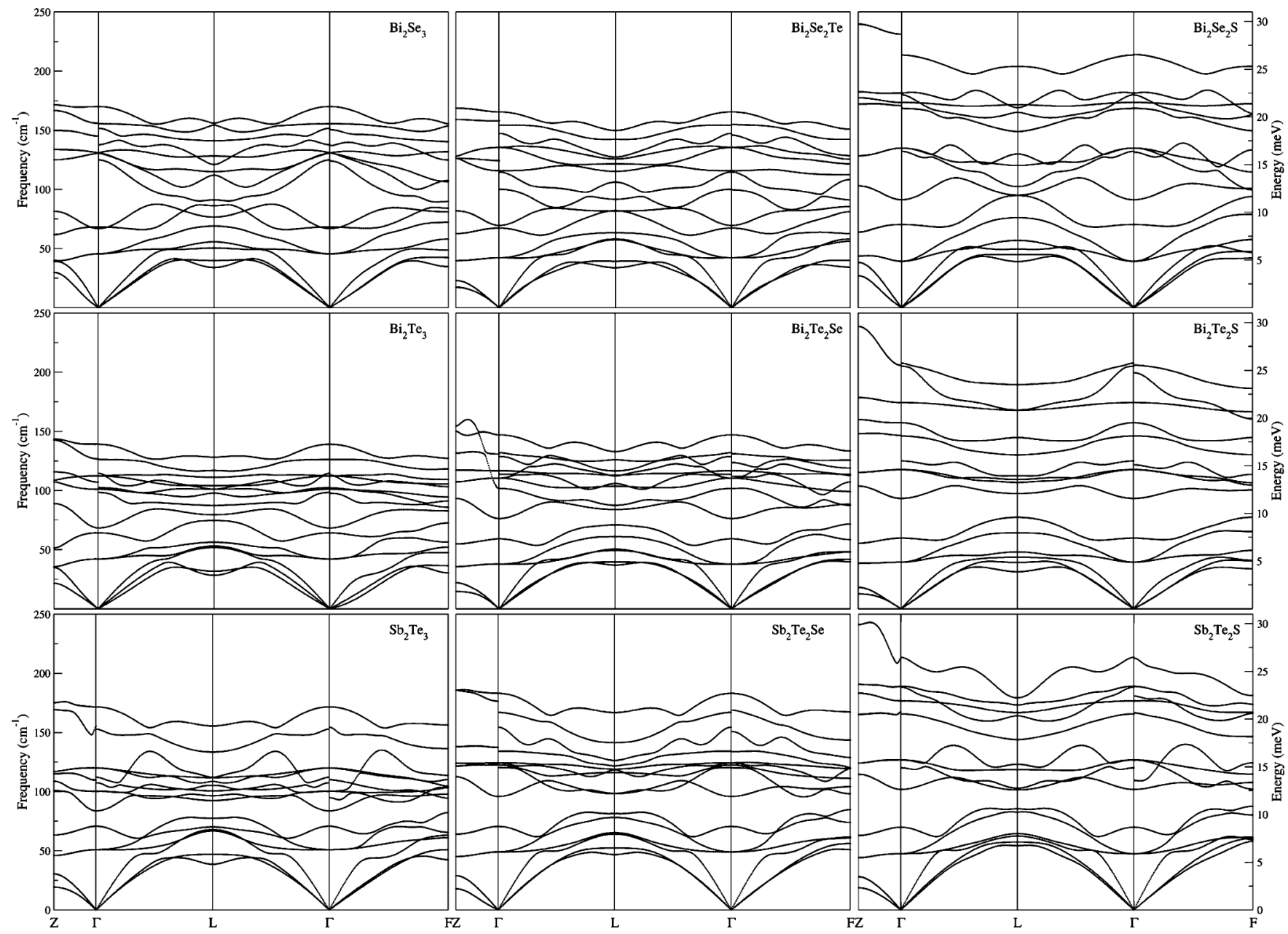

FIG. 4. Phonon dispersion curves along the high symmetry directions of the Brillouin zone of the $\mathrm{V}_{2} \mathrm{VI}_{3}$ bulk crystals. (Top row) $\mathrm{Bi}_{2} \mathrm{Se}_{3}$, $\mathrm{Bi}_{2} \mathrm{Se}_{2} \mathrm{Te}$, and $\mathrm{Bi}_{2} \mathrm{Se}_{2} \mathrm{~S}$. (Middle row) $\mathrm{Bi}_{2} \mathrm{Te}_{3}, \mathrm{Bi}_{2} \mathrm{Te}_{2} \mathrm{Se}$, and $\mathrm{Bi}_{2} \mathrm{Te}_{2} \mathrm{~S}$. (Bottom row) $\mathrm{Sb}_{2} \mathrm{Te}_{3}, \mathrm{Sb}_{2} \mathrm{Te}_{2} \mathrm{Se}_{2}$, and $\mathrm{Sb}_{2} \mathrm{Te}_{2} \mathrm{~S}$.

mode is found at $45.6 \mathrm{~cm}^{-1}$ at the $\Gamma$ point, $49.8 \mathrm{~cm}^{-1}$ at the $L$ point, $48.6 \mathrm{~cm}^{-1}$ at the $F$ point, and $39.5 \mathrm{~cm}^{-1}$ at the $Z$ point. Along the $\Gamma-Z$ direction the mode becomes softer, which holds for other compounds except for $\mathrm{Bi}_{2} \mathrm{Se}_{2} \mathrm{~S}$ where the mode has a lower frequency at $\Gamma$ compared to the frequency at the $Z$ point. In several cases the mode experiences a stiffening at the $L$ point where it interacts with some acoustic modes and exhibits an avoided crossing. See, for example, the dispersion curves of $\mathrm{Bi}_{2} \mathrm{Se}_{2} \mathrm{Te}, \mathrm{Bi}_{2} \mathrm{Te}_{3}$, and $\mathrm{Sb}_{2} \mathrm{Te}_{3}$ (Fig. 4) around the $F$ point.

The second Raman active mode $A_{1 g}^{1}$ is shown in Fig. 5(b) where the atoms are displaced towards each other along the trigonal axis and the middle atom within the QLs being at rest. The ordering of the frequency for the $A_{1 g}^{1}$ mode is found to be the same as that of the $E_{g}^{1}$ mode. The same ordering of the frequency is found for the ternary compounds as well.

When the binary compound of $\mathrm{Bi}_{2} \mathrm{Te}_{3}$ is compared to its ternary derivatives, $\mathrm{Bi}_{2} \mathrm{Te}_{2} \mathrm{Se}$ and $\mathrm{Bi}_{2} \mathrm{Te}_{2} \mathrm{~S}$, one notices that the frequency for the $\mathrm{Bi}_{2} \mathrm{Te}_{3}$ is larger than for the ternary ones. Our explanation for this, which is applied as well for the other compounds, is that the Bi-Te layers in the bulk QLs is more decoupled from the Te middle layer in the QLs while the internal bond length between $\mathrm{Bi}-\mathrm{Te}$ is somewhat shorter than in the ternary compounds. In comparison with the $E_{g}^{1}$ mode the $A_{1 g}^{1}$ mode acquires more dispersion throughout the $\mathrm{BZ}$ and exhibits several avoided crossings with other phonon modes that have similar symmetry.

The third Raman active mode $E_{g}^{2}$ [Fig. 5(e)] corresponds to in-phase displacements of the atoms. This mode turns out to have a more complex behavior in regard to the ordering of the frequency for the different compounds. $\mathrm{Bi}_{2} \mathrm{Te}_{3}$ has the lowest frequency for the $E_{g}^{2}$ mode followed by the second lowest that corresponds to $\mathrm{Sb}_{2} \mathrm{Te}_{3}$ and finally $\mathrm{Bi}_{2} \mathrm{Se}_{3}$, which has the highest frequency. The difference in the order compared to the former described modes can be partially understood by examining the displacement field of the mode [Fig. 5(e)]. The displacement field of the $E_{g}^{2}$ mode shows that the Te atoms in $\mathrm{Bi}_{2} \mathrm{Te}_{3}$ and $\mathrm{Sb}_{2} \mathrm{Te}_{3}$, respectively, and the Se atoms in $\mathrm{Bi}_{2} \mathrm{Se}_{3}$ have larger displacements than the $\mathrm{Bi}$ and $\mathrm{Sb}$ atoms which would explain the ordering in the phonon mode frequency when the mass of the atoms is taken into account.

In the same way one can understand the reordering of the $E_{g}^{2}$ mode with respect to the compound in question as well as for the ternary compounds. Here the ordering of the $E_{g}^{2}$ mode is as follow, $\mathrm{Bi}_{2} \mathrm{Te}_{2} \mathrm{Se}$ having the lowest $E_{g}^{2}$ frequency followed by $\mathrm{Bi}_{2} \mathrm{Se}_{2} \mathrm{Te}, \mathrm{Bi}_{2} \mathrm{Te}_{2} \mathrm{~S}, \mathrm{Sb}_{2} \mathrm{Te}_{2} \mathrm{Se}, \mathrm{Sb}_{2} \mathrm{Te}_{2} \mathrm{~S}$, and $\mathrm{Bi}_{2} \mathrm{Se}_{2} \mathrm{~S}$, 
TABLE II. Calculated and experimental frequencies at the BZ $\bar{\Gamma}$ point.

\begin{tabular}{|c|c|c|c|c|c|c|c|c|}
\hline & \multicolumn{2}{|c|}{$E_{g}^{1}\left(\mathrm{~cm}^{-1}\right)$} & \multicolumn{2}{|c|}{$A_{1 g}^{1}\left(\mathrm{~cm}^{-1}\right)$} & \multicolumn{2}{|c|}{$E_{g}^{2}\left(\mathrm{~cm}^{-1}\right)$} & \multicolumn{2}{|c|}{$A_{1 g}^{2}\left(\mathrm{~cm}^{-1}\right)$} \\
\hline & Expt. & Theory & Expt. & Theory & Expt. & Theory & Expt. & Theory \\
\hline $\mathrm{Bi}_{2} \mathrm{Se}_{3}$ & & 45.6 & 72.5 & 68.3 & 131.5 & 130.7 & 176.5 & 170.1 \\
\hline $\mathrm{Bi}_{2} \mathrm{Te}_{3}$ & & 42.1 & 61.5 & 64.2 & 101.5 & 112.3 & 133.5 & 139.2 \\
\hline $\mathrm{Sb}_{2} \mathrm{Te}_{3}$ & & 50.8 & 68.0 & 69.2 & 111.0 & 120.0 & 164.5 & 171.4 \\
\hline $\mathrm{Bi}_{2} \mathrm{Se}_{2} \mathrm{~S}$ & & 45.1 & & 69.4 & & 133.5 & & 180.8 \\
\hline $\mathrm{Bi}_{2} \mathrm{Te}_{2} \mathrm{~S}$ & & 39.5 & 62.0 & 59.7 & 104.5 & 117.7 & 148.5 & 157.4 \\
\hline $\mathrm{Bi}_{2} \mathrm{Te}_{2} \mathrm{Se}$ & & 38.2 & 62.0 & 58.9 & 103.5 & 110.5 & 144.5 & 146.7 \\
\hline $\mathrm{Bi}_{2} \mathrm{Se}_{2} \mathrm{Te}$ & & 42.2 & 65.5 & 67.4 & 110.0 & 115.7 & 159.0 & 165.5 \\
\hline $\mathrm{Sb}_{2} \mathrm{Te}_{2} \mathrm{Se}$ & 35.0 & 49.0 & 62.5 & 70.6 & 106.5 & 124.3 & 168.0 & 183.1 \\
\hline $\mathrm{Sb}_{2} \mathrm{Te}_{2} \mathrm{~S}$ & & 47.5 & 69.0 & 69.9 & 112.0 & 126.7 & 168.0 & 188.9 \\
\hline
\end{tabular}

respectively. One can then notice that the ordering of the $E_{g}^{2}$ mode with respect to the compound behaves quite nicely when one takes into account which atoms have a larger displacement and as well the atomic mass of that atom. Overall the agreement with the experimental Raman spectroscopy is very good. The $E_{g}^{2}$ mode is a double degenerate mode at the BZ $\Gamma$ point. Away from the $\Gamma$ point, in several cases, one branch of the $E_{g}^{2}$ mode disperses throughout the BZ and interacts with other modes while the other phonon branch remains almost dispersionless.

The fourth Raman active mode corresponds to the $A_{1 g}^{2}$ mode that is characterized by an in-phase displacement of the atoms involved [Fig. 5(h)]. For the $A_{1 g}^{2}$ mode the ordering of the frequency is restored to that of the $E_{g}^{1}$ mode both for the binary compounds and for the ternary compounds. The agreement with the experimental results is very good.
The displacement field of the $A_{1 g}^{2}$ mode does not involve the edge atoms in the rhombohedral unit cell and in all cases except when the $\mathrm{S}$ atom occupies that position the $A_{1 g}^{2}$ mode has the highest frequency among all other phonon modes. Throughout the BZ the mode acquires some dispersion as well as some interaction with other modes.

\section{Dynamical properties of one $Q L$}

Phonon dispersion curves for one quintuple layer of the binary and ternary compounds have been calculated and the results are presented in Fig. 6. For one $\mathrm{QL}$ of $\mathrm{Bi}_{2} \mathrm{Se}_{3}$ also the spin-orbit interaction has been taken into account. A summary of the Raman active modes at the surface Brillouin zone $\bar{\Gamma}$ point is presented in Table III. Overall the Raman active modes of the QLs are close to the calculated bulk Raman modes. (a)

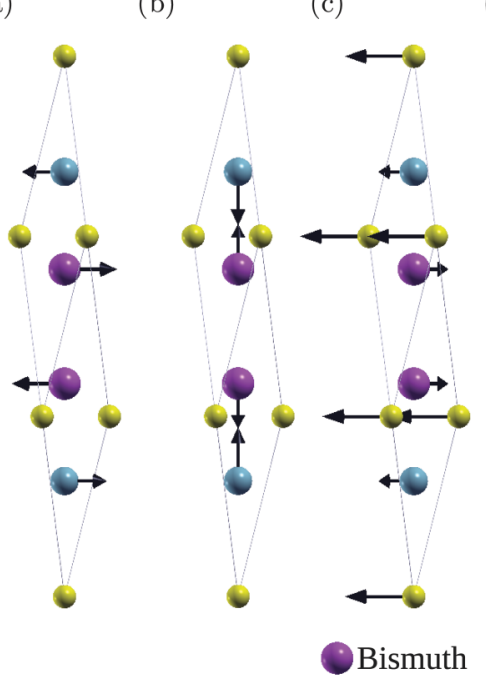

(d)

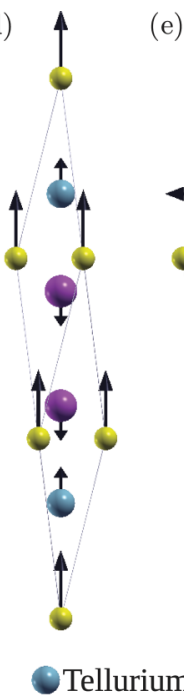

e)

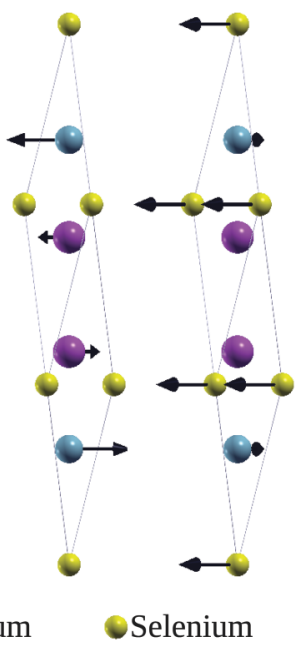

(g)

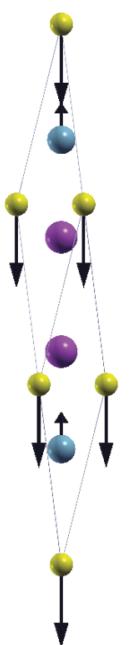

(h)

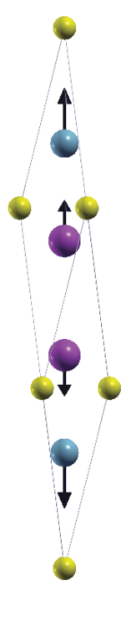

FIG. 5. (Color online) Phonon displacement patterns for a crystal with rhombohedral symmetry $\left(D_{3 d}^{5}, R \overline{3} m\right)$. Phonons at the $\Gamma$ point are classified accordingly: $\Gamma=2\left(A_{1 g}+E_{g}\right)+3\left(E_{u}+A_{2 u}\right)$, where two $E_{u}$ and one $A_{2 u}$ are acoustic modes, respectively. The displacement patterns are displayed from a side view where the rhombohedral unit cell has been rotated slightly in order to facilitate the view of the displacement vectors. In the figure (a) corresponds to the double degenerate Raman active $E_{g}^{1}$ mode, (b) the Raman active $A_{1 g}^{1}$ mode, (c) the double degenerate infrared active $E_{u}$ mode, (d) the infrared active $A_{2 u}$ mode, (e) the double degenerate Raman active $E_{g}^{2}$ mode, (f) the double degenerate infrared active $E_{u}$ mode, (g) the infrared active $A_{2 u}$ mode, and (h) the Raman active $A_{1 g}^{2}$ mode, respectively. The rhombohedral unit cell would in this case correspond to a ternary compound, say $\mathrm{Bi}_{2} \mathrm{Te}_{2} \mathrm{Se}$, where the $\mathrm{Bi}$ atom have the largest radius (magenta) followed by the Te atom (cyan) and the Se atoms (yellow) that occupy the edges of the rhombohedral unit cell. 


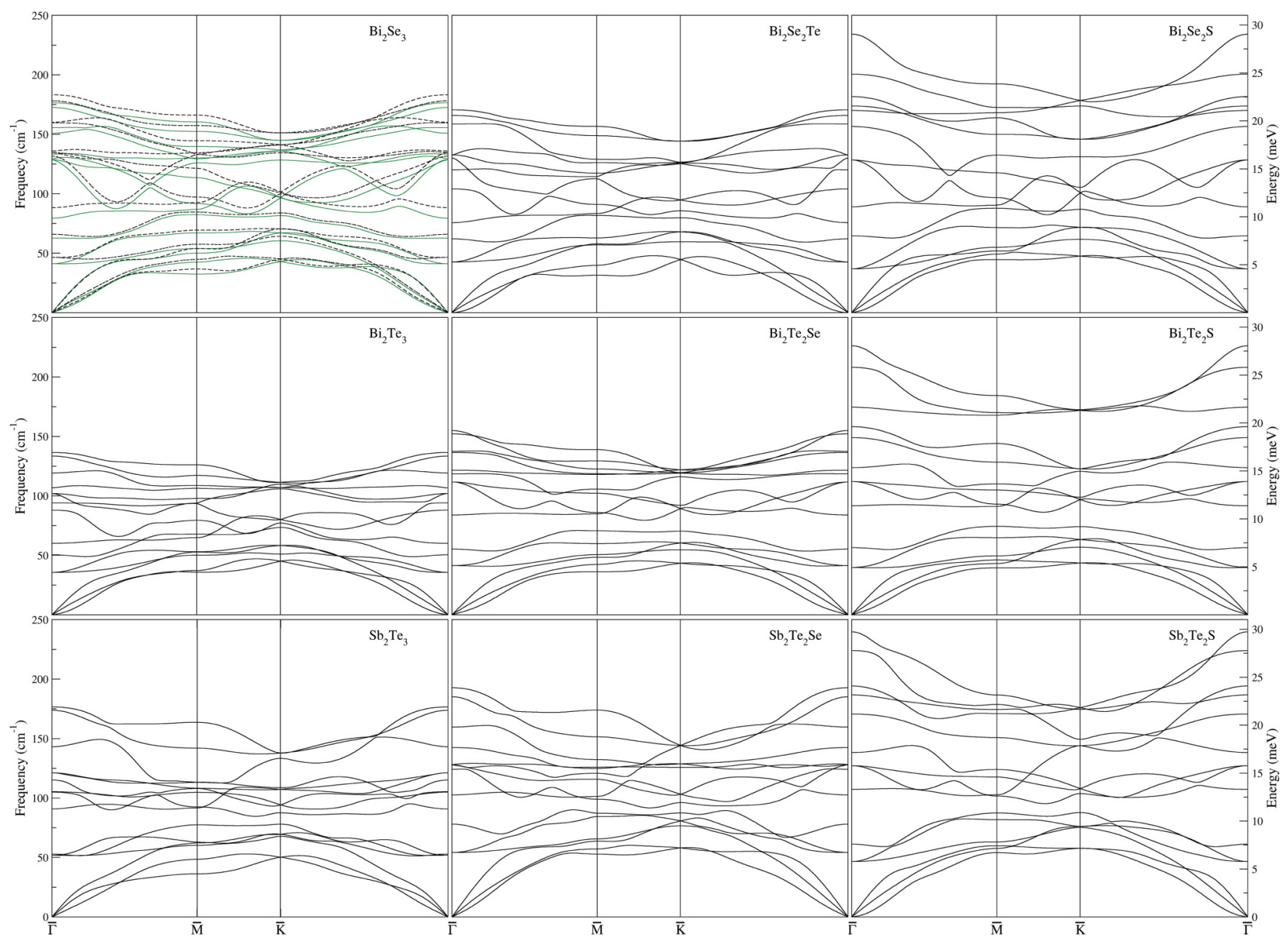

FIG. 6. (Color online) Phonon dispersion curves for the binary and ternary compounds shown along the high symmetry directions of the irreducible surface Brillouin zone for one QL. In the upper left figure we show also the phonon dispersion curves for both spin-orbit calculations (solid lines) and as well for non-spin-orbit calculations (dashed lines).

The $E_{g}^{1}$ mode becomes somewhat softer for certain compounds while for others it becomes stiffer, however, the shift in the $E_{g}^{1}$-mode frequency for the different compounds is not dramatic. On the other hand the $A_{1 g}^{1}$ mode is softer in all compounds except for one QL of $\mathrm{Sb}_{2} \mathrm{Te}_{2} \mathrm{Se}$ where it is stiffer

TABLE III. Energy and character of the $\bar{\Gamma}$-point Raman active phonon modes of the optimized geometry at the theoretical equilibrium lattice parameters for one $\mathrm{QL}$.

\begin{tabular}{lcccc}
\hline \hline $1 \mathrm{QL}$ & $E_{g}^{1}\left(\mathrm{~cm}^{-1}\right)$ & $A_{1 g}^{1}\left(\mathrm{~cm}^{-1}\right)$ & $E_{g}^{2}\left(\mathrm{~cm}^{-1}\right)$ & $A_{1 g}^{2}\left(\mathrm{~cm}^{-1}\right)$ \\
\hline $\mathrm{Bi}_{2} \mathrm{Se}_{3}$ & 46.5 & 66.0 & 134.6 & 183.3 \\
$\mathrm{Bi}_{2} \mathrm{Te}_{3}$ & 36.0 & 50.5 & 102.0 & 136.5 \\
$\mathrm{Sb}_{2} \mathrm{Te}_{3}$ & 51.8 & 52.9 & 121.3 & 176.6 \\
$\mathrm{Bi}_{2} \mathrm{Se}_{2} \mathrm{~S}$ & 43.1 & 60.8 & 128.4 & 181.7 \\
$\mathrm{Bi}_{2} \mathrm{Te}_{2} \mathrm{~S}$ & 39.7 & 56.5 & 112.0 & 158.2 \\
$\mathrm{Bi}_{2} \mathrm{Te}_{2} \mathrm{Se}$ & 41.4 & 55.3 & 111.7 & 152.2 \\
$\mathrm{Bi}_{2} \mathrm{Se}_{2} \mathrm{Te}$ & 42.5 & 62.0 & 132.5 & 170.5 \\
$\mathrm{Sb}_{2} \mathrm{Te}_{2} \mathrm{Se}$ & 54.2 & 78.1 & 128.0 & 192.8 \\
$\mathrm{Sb}_{2} \mathrm{Te}_{2} \mathrm{~S}$ & 46.6 & 61.2 & 127.2 & 194.2 \\
\hline \hline
\end{tabular}

by about $8 \mathrm{~cm}^{-1}$. The $E_{g}^{2}$-mode frequency agrees very well with the bulk calculated frequencies where the difference is not large. In the same fashion as in the bulk case one QL of $\mathrm{Bi}_{2} \mathrm{Se}_{2} \mathrm{Te}$ gives a $E_{g}^{2}$-mode frequency that is much larger than the experimental one. The $A_{1 g}^{2}$ mode of the QL changes very little for most of the materials as can be compared with the values in Table II. For the antimony based ternary materials the QL $A_{1 g}^{2}$ mode shifts to higher frequencies.

The dispersion curves of one QL look very similar to each other. The ternary compounds that include $\mathrm{S}$ atoms in the middle of the QL exhibit the highest phonon frequency maxima and in the case of $\mathrm{Bi}_{2} \mathrm{Te}_{2} \mathrm{~S}$ there is a clear demarcation between the three topmost phonon branches and the remaining phonon branches. These branches correspond to pure $\mathrm{S}$ vibrations, namely one with longitudinal (L), one shear-vertical (SV), and one shear-horizontal ( $\mathrm{SH}$ ) polarization, respectively.

The Rayleigh mode for single crystal surfaces in general has the largest amplitude on the topmost atomic layer and the polarization is shear vertical. However, it is found that for layered compounds such as $\mathrm{Bi}_{2} \mathrm{Se}_{3}$ and other compositions studied the Rayleigh mode is confined to the second layer of the ultrathin film surface. The only exception is for one QL of 

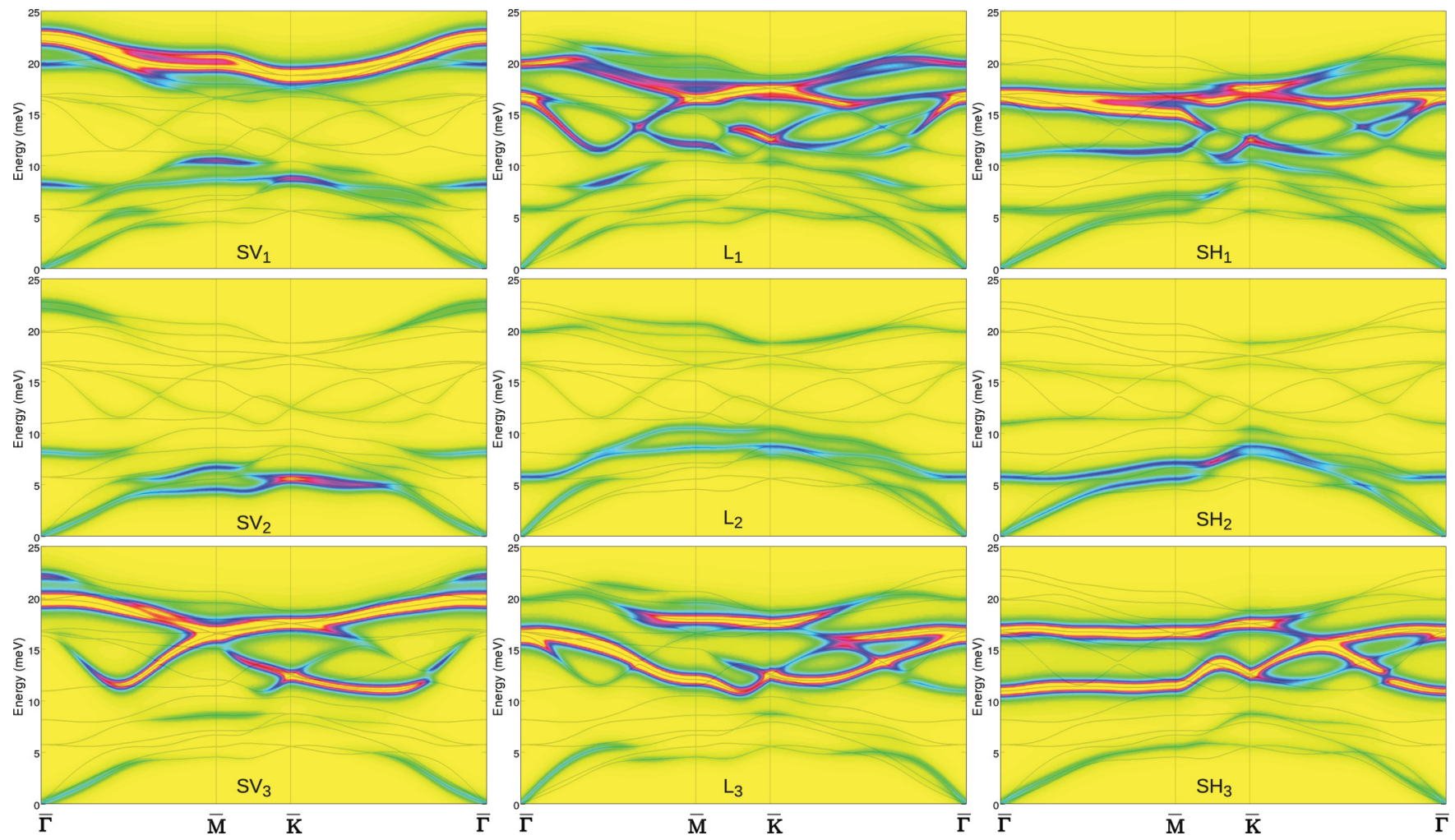

FIG. 7. (Color online) Contour plots of the phonon density of states projected onto the first (Se), second (Bi), and third (Se) QL layer for shear-vertical $\left(\mathrm{SV}_{i}\right)$, longitudinal $\left(\mathrm{L}_{i}\right)$, and shear-horizontal $\left(\mathrm{SH}_{i}\right)$ polarizations; $i$ corresponds to the layer index.

$\mathrm{Sb}_{2} \mathrm{Te}_{3}$ where the shear-vertical mode has Sb displacements in the vicinity of the $\bar{\Gamma}$ point and towards the $\bar{M}$ point the lowest mode acquires Te displacements. One would expect a similar behavior also for the QLs of $\mathrm{Sb}_{2} \mathrm{Te}_{2} \mathrm{Se}$ and $\mathrm{Sb}_{2} \mathrm{Te}_{2} \mathrm{~S}$ where the lowest mode now has purely antimony SV character, that is, second atomic layer displacements. This behavior is also seen in the $\mathrm{Bi}_{2} \mathrm{Se}_{3} \mathrm{QL}$ and will be analyzed in detail below.

\section{One $\mathrm{QL}$ of $\mathrm{Bi}_{2} \mathrm{Se}_{3}$}

The dynamical properties of one $\mathrm{QL}$ of $\mathrm{Bi}_{2} \mathrm{Se}_{3}$ were calculated with and without the inclusion of spin-orbit interaction. The phonon dispersions are shown in Fig. 6 where the scalar relativistic results are shown as dashed lines and the fully relativistic results as solid lines, respectively.

In general, the inclusion of spin-orbit interaction results in a rigid shift of the phonon bands to lower energies. However, the softening is larger for the high-energy phonon branches at about $10 \mathrm{meV}$ and up to maximum phonon energy while for the lower energy phonon branches the softening is less pronounced. The largest shift in the branches amounts to the order of $\sim 1 \mathrm{meV}$. The constant shift of the upper branches originates from the somewhat extended separation between the topmost Se layer and the underlying Bi layer for the calculations including the spin-orbit interaction, while for the lower phonon branches where the energy shift is less pronounced, the difference in the interlayer spacing between $\mathrm{Bi}$ and the inner Se layer is much smaller. It is worth noting that a similar effect of spin-orbit interaction, namely a decrease of frequencies when spin-orbit interaction is included has also been reported for pristine bulk $\mathrm{Pb}^{43}$ and $\mathrm{Bi}^{44}$ as well as for $\mathrm{BiTeCl}$ and BiTeI compounds. ${ }^{45}$

For a closer analysis we present in Fig. 7 the polarization and layer projected phonon density of states (Ph-DOS) for a QL where SO interaction is not included. The Ph-DOS for a QL with the inclusion of SO interaction is basically identical and is not shown. The QL slab is constructed in such a way that it has inversion symmetry with respect to the central layer and thus we display only the Ph-DOS corresponding to the two uppermost atomic layers and the center layer. The Ph-DOS are shown for all possible polarizations indicated in the figure as $\mathrm{SV}_{i}, \mathrm{~L}_{i}$, and $\mathrm{SH}_{i}$, where $i=1,2,3$ corresponds to projection onto the corresponding atomic layer.

In Fig. 7, we see an intense nondegenerate optical phonon mode, $\mathrm{SV}_{1}$, belonging to the topmost Se layer; the mode has the highest phonon energy within the surface Brillouin zone (SBZ) compared to any other phonon modes. It is nondegenerate for the most part of the SBZ due to interaction through the QL between modes that belong to the opposite surface of the slab. At $\bar{\Gamma}$ the mode has an energy of $22.4 \mathrm{meV}$. Around the $\bar{\Gamma}$ point the displacement field of the mode incorporates all the layers within the $\mathrm{QL}$ as seen in the figure $\left(\mathrm{SV}_{1}, \mathrm{SV}_{2}\right.$, and $\mathrm{SV}_{3}$ ), although it has the strongest intensity in the topmost layer. At and around the SBZ $\overline{\mathrm{K}}$ point this mode becomes degenerate with the second layer mode that is longitudinal in character $\left(\mathrm{L}_{2}\right)$. Figure 7 also shows several other modes that have a coupling to the topmost Se layer. These modes are lower in energy and are distributed over several atomic layers. One such mode is the $8.2-\mathrm{meV}$ mode at $\bar{\Gamma}$ which involves the displacement of the topmost Se layer $\left(\mathrm{SV}_{1}\right)$ and as well the 


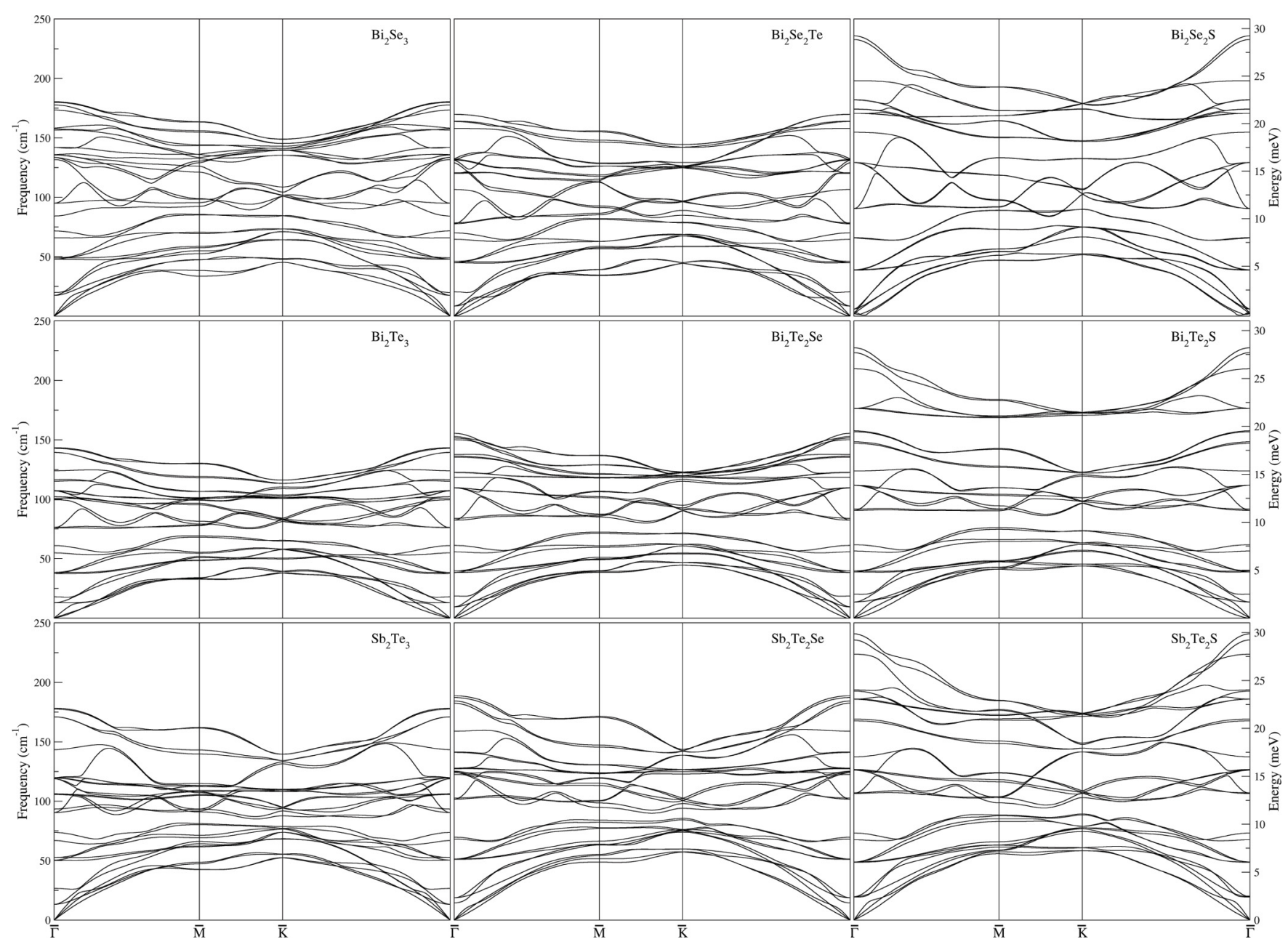

FIG. 8. Phonon dispersion curves for the binary and ternary compounds shown along the high symmetry directions of the irreducible surface Brillouin zone for two QLs.

underlying Bi layer $\left(\mathrm{SV}_{2}\right)$. This mode persists about halfway from the $\overline{\bar{\Gamma}}$ to $\overline{\mathrm{M}}$ and halfway from $\bar{\Gamma}$ to $\overline{\mathrm{K}}$ before an avoided crossing and hybridization with other modes takes place.

The acoustic modes of the QL are ordered as follows from the lowest to the highest energy along the SBZ symmetry directions. The SV mode has strongest intensity in the second atomic layer and with an energy of $4.6 \mathrm{meV}$ at the $\overline{\mathrm{M}}$ point and $5.4 \mathrm{meV}$ at the $\overline{\mathrm{K}}$ point. With the second lowest energy of the acoustic modes is an SH mode that as well as the SV mode has the highest intensity in the Bi layer (i.e., $\mathrm{SH}_{2}$ ). The energy of the mode at high symmetry points of the SBZ are $5.6 \mathrm{meV}(\overline{\mathrm{M}}$ point) and $8.0 \mathrm{meV}$ ( $\overline{\mathrm{K}}$ point), respectively. The third acoustic mode is the one with $\mathrm{L}$ character. The mode has strongest intensity on the Bi layer $\left(\mathrm{L}_{2}\right)$ and some intensity on the Se layers $\left(\mathrm{L}_{1}\right.$ and $\left.\mathrm{L}_{3}\right)$. Along the $\bar{\Gamma}-\overline{\mathrm{M}}$ and $\bar{\Gamma}-\overline{\mathrm{K}}$ directions the $\mathrm{L}$ acoustic mode encounters an avoided crossing with another $\mathrm{L}$ mode that is an optical mode. After the avoided crossing the mode persists up to the $\bar{M}$ point $(10.5 \mathrm{meV})$ where it hybridizes with an SV mode and acquires some intensity in the first layer $\left(\mathrm{SV}_{1}\right)$. The evolution of the mode is very similar to that of the longitudinal resonance of the $\mathrm{Cu}(111)$ surface. ${ }^{46,47}$ The mentioned L optical mode undergoes a very similar scenario as described above. In the $\bar{\Gamma}$ point where the mode forms a degenerate pair with a shear-horizontal mode the mode has an energy of $5.8 \mathrm{meV}$ and at the $\overline{\mathrm{M}}$ point $8.6 \mathrm{meV}$. In addition we find other modes that have $\mathrm{L}_{i}$ and $\mathrm{SH}_{i}$ polarization and are degenerate at the $\bar{\Gamma}$ point; namely the $\mathrm{L}_{1}$ and $\mathrm{L}_{3}$ modes form degenerate pairs with $\mathrm{SH}_{1}$ and $\mathrm{SH}_{3}$ at $16.7 \mathrm{meV}$ and $11.0 \mathrm{meV}$, respectively.

\section{Dynamical properties of two QLs}

In Fig. 8 we present the calculated phonon dispersion curves for two QLs. The phonon dispersion curves for the two QLs are very similar to the dispersion curves for the one $\mathrm{QL}$, except that in the case of the two QLs we now have additional modes due to the increased number of atoms. In regard to the Raman active modes described in the above sections one can see that there is not much difference between one QL and two QLs and the frequencies of the modes can be directly compared with those corresponding to one QL.

However, there are some modes and features that arise due to the effect of including an additional QL. These additional phonon modes are illustrated in Fig. 9 for two QLs of $\mathrm{Sb}_{2} \mathrm{Te}_{3}$. In Fig. 9(a) the displacement field corresponds to a phonon mode with $13.17 \mathrm{~cm}^{-1}$ at the SBZ $\bar{\Gamma}$ point. The mode is double degenerate at the $\bar{\Gamma}$ point and is characterized by a shear displacement of all QLs in the out-of-phase motion. The 
(a)

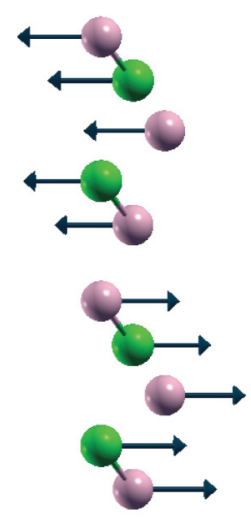

(b)

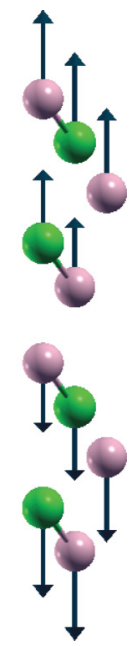

FIG. 9. (Color online) $\bar{\Gamma}$-point phonon displacement pattern for two QLs of $\mathrm{Sb}_{2} \mathrm{Te}_{3}$. Panel (a) corresponds to a horizontal shear mode $\left(13.17 \mathrm{~cm}^{-1}\right)$ and $(b)$ to the shear-vertical QL mode $\left(26.63 \mathrm{~cm}^{-1}\right)$.

second mode illustrated in Fig. 9(b) corresponds to a phonon mode with a frequency of $26.63 \mathrm{~cm}^{-1}$ at the SBZ $\bar{\Gamma}$ point. The mode is characterized by a shear-vertical symmetrical displacement. The antisymmetric mode would correspond to a rigid displacement of all QLs and is considered as an acoustic mode. We consider two QLs of $\mathrm{Sb}_{2} \mathrm{Te}_{3}$ since the modes are easy to notice in the phonon dispersion curves and are well separated from each other. That is not always the case and

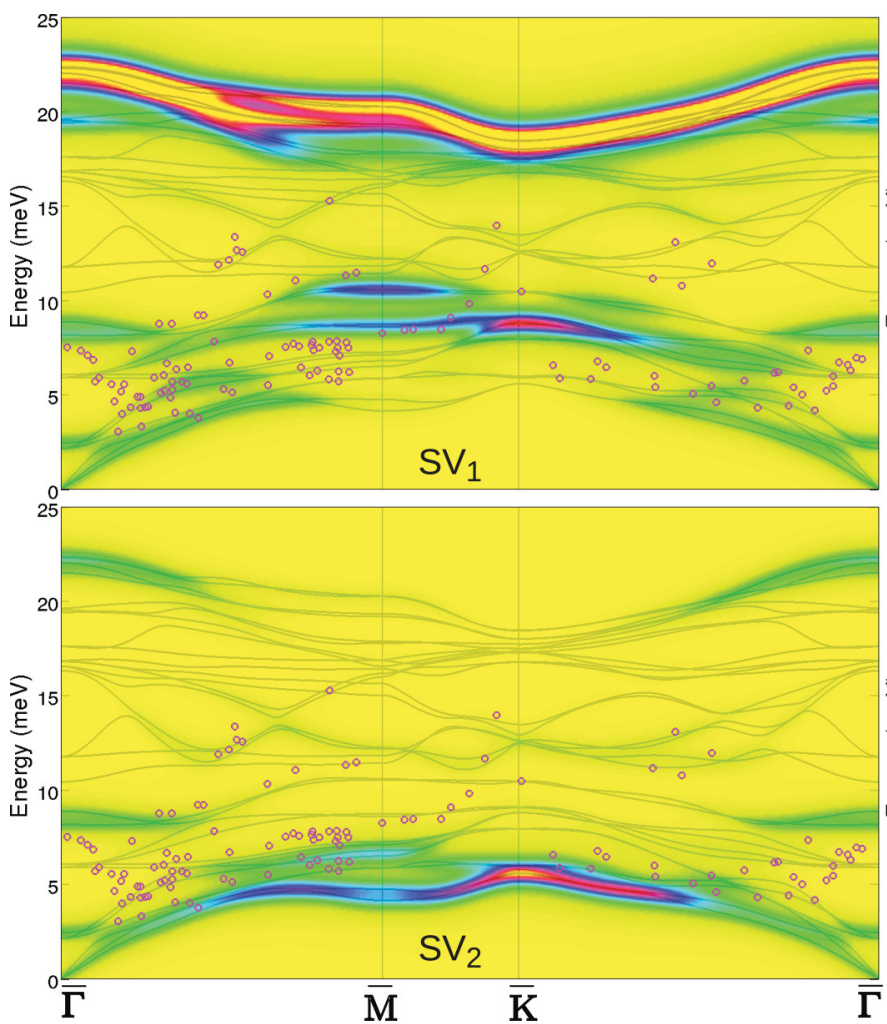

for some compounds the two (three, counting the degenerate mode) modes are almost degenerate, for example, two QLs of $\mathrm{Sb}_{2} \mathrm{Te}_{2} \mathrm{~S}$ and $\mathrm{Bi}_{2} \mathrm{Se}_{3}$ that are nearly degenerate.

\section{A. Two QLs of $\mathrm{Bi}_{2} \mathrm{Se}_{3}$ and comparison to experimental data}

In a very recent letter ${ }^{27}$ experimental elastic and inelastic helium atom scattering (HAS) results were reported for the (001) surface of $\mathrm{Bi}_{2} \mathrm{Se}_{3}$ together with calculations based on the phenomenological pseudocharge model. ${ }^{48}$ The HAS results together with the calculations reported on a strong Kohn anomaly showing up in the phonon dispersion curves as a strong dip at about $0.2 \AA^{-1}$ from the $\bar{\Gamma}$ point. In addition, HAS surface phonon dispersion curves along the SBZ were reported. The He atoms are scattered by the phonon induced charge density oscillations at about 2-3 $\AA$ outside the surface which makes HAS experiments very sensitive to changes in the surface charge and one can be assured that the reported scattering intensities are surface phonon modes. In this section we will not attempt to describe the reported Kohn anomaly but we will address the similarities between our first-principles calculations for the ultrathin $\mathrm{Bi}_{2} \mathrm{Se}_{3}$ QLs and the reported HAS phonon dispersion curves.

As previously reported, ${ }^{46,47,49}$ HAS can detect surface modes and surface resonances as well as phonon modes that belong to deeper layers that give rise to induced charge density oscillations in the region of the He atom turning point. With that in mind, we present in Fig. 10 the Ph-DOS projected onto the first and second layer with L or SV polarizations together

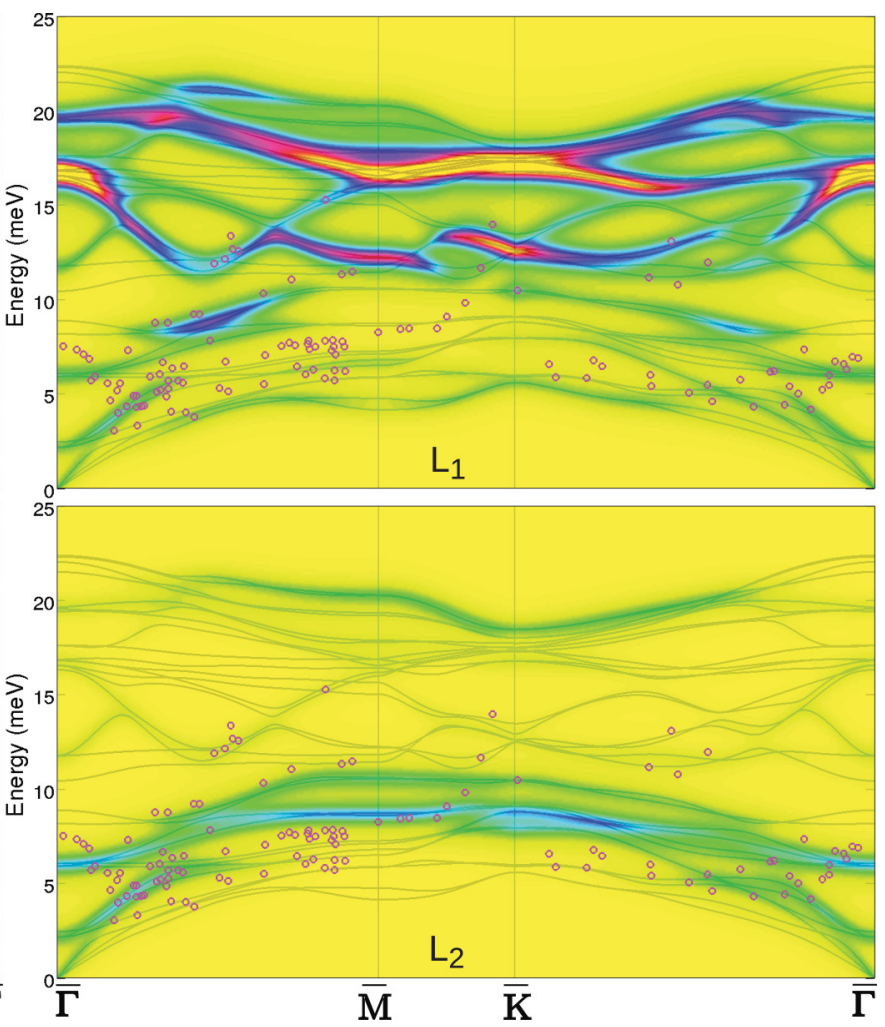

FIG. 10. (Color online) Contour plots of the phonon density of states projected onto the first (Se), second (Bi), and third (Se) layers of the two-QLs system for shear-vertical $\left(\mathrm{SV}_{i}\right)$, longitudinal $\left(\mathrm{L}_{i}\right)$, and shear-horizontal $\left(\mathrm{SH}_{i}\right)$ polarizations; $i$ corresponds to the layer index. The experimental data ${ }^{27}$ points are shown as open circles. 
with the extracted experimental results. As described in the above sections, surface modes that correspond to the topmost Se layer belong mostly to the top part of the phonon energy spectra although there are some signatures in the lower part of the energy spectra where most of the phonon branches have strong signature of the bismuth layer. It is fair to say that most of the HAS data points correspond to phonon branches that lie in the second layer, for example, bismuth layer. In the figure the experimental data look very scattered at shorter wavelengths while towards the high symmetry points the data becomes more coherent which makes easier the comparison with our calculations.

The data points in the lower energy part seem to follow nicely the longitudinal acoustic phonon mode up to about midway along the $\bar{\Gamma}-\bar{M}$ direction where the continuation of this mode has $\mathrm{SV}$ character in the second layer $\left(\mathrm{SV}_{2}\right)$. While in the $\bar{\Gamma}-\overline{\mathrm{K}}$ direction the data points can be referred to either the longitudinal acoustic mode or the longitudinal optic mode and close to the $\overline{\mathrm{K}}$ point the mode transforms into an $\mathrm{SV}_{2}$ character. In general we see that most of the calculated modes in this paper have somewhat lower energy than the reported experimental data. This might be due to the fact that we analyze an ultrathin film consisting of only two QLs whereas the HAS experiments are performed on a semi-infinite surface. Bearing that in mind, several data points towards the $\bar{M}$ point can be assigned. In the $\bar{\Gamma}-\overline{\mathrm{K}}$ direction we have the longitudinal mode $\left(\mathrm{L}_{2}\right)$ that undergoes an avoided crossing with the $\mathrm{L}_{2}$ optical mode. The natural continuation for the acoustic mode follows that of the $\mathrm{SV}_{2}$ mode towards the $\overline{\mathrm{M}}$ point. On the other hand the acoustic $L_{2}$ mode reappears after the avoided crossing with the optical $\mathrm{L}_{2}$ mode and at the $\overline{\mathrm{M}}$ point it has an energy of $10.4 \mathrm{meV}$. The data points follow both the described branches rather well.

\section{CONCLUSIONS}

Raman spectroscopy and density-functional perturbation theory calculations have been applied to study the vibrational properties of binary and ternary $\mathrm{V}_{2} \mathrm{VI}_{3}$ compounds that are classified as topological insulators. The overall agreement between theory and experiments is very good and from theory we are able to identify, analyze, and characterize the experimen- tally detected Raman active modes. The dispersion curves of several Raman active modes within the Brillouin zone show a rather flat dispersion. Phonon modes of the ternary compounds that include sulfur in the composition experience the highest phonon energy within the Brillouin zone. $\mathrm{Sb}_{2} \mathrm{Te}_{2} \mathrm{~S}$ have the highest maximum phonon energy while $\mathrm{Bi}_{2} \mathrm{Te}_{3}$ have the lowest maximum phonon energy. The phonon dispersion curves of ultrathin films (one and two QLs) of the binary and ternary compounds were calculated as well. For one $\mathrm{QL}$ of $\mathrm{Bi}_{2} \mathrm{Se}_{3}$ we show that the inclusion of spin-orbit interaction has little influence on the phonon frequencies and the character of the phonon modes are preserved. When more than one $\mathrm{QL}$ is taken into account new modes appear that are originating from displacements of entire QL blocks. One can expect a series of new modes as the number of QL blocks taken into account increases.

Despite the fact two QLs of $\mathrm{Bi}_{2} \mathrm{Se}_{3}$ still form an insulator, it is interesting to compare the present results with the recent inelastic He atom scattering data for the semiinfinite crystal, which is metallic with a Dirac cone at $\bar{\Gamma}$. HAS, unlike electron energy loss spectroscopy, can probe phonons localized on deep layers beneath the surface via the electron-phonon coupling. ${ }^{46,47,49,50}$ Indeed the comparison of Zhu et al. HAS data, reproduced in Fig. 10, with the calculated phonon densities of $\mathrm{L}$ and $\mathrm{SV}$ polarizations projected onto the first ( $\mathrm{Se}$ ) and second (Bi) layers, clearly shows that the RW has the largest amplitude in the second layer $\left(\mathrm{SV}_{2}\right)$. In addition, the mode at $5.5 \mathrm{meV}$ ( $\bar{\Gamma}$ point $)$, which in HAS data is indicated as anomalous, shows in the calculation the largest amplitude in the second layer $\left(\mathrm{L}_{2}\right)$ along $\bar{\Gamma}-\bar{K}$. The large $\mathrm{RW}$ amplitude on the second layer is also found in $\mathrm{Sb}(111)^{51}$ and $\mathrm{Bi}(111){ }^{52}$

\section{ACKNOWLEDGMENTS}

K.A.K. and O.E.T. acknowledge financial support by the RSSF and RFBR (Grant No. 12-02-00226). All equipment belongs to Scientific and Educational Center "Nanosystems and Advance Materials" of Novosibirsk State University. We acknowledge partial support from the University of the Basque Country (Project No. GV-UPV/EHU and Grant No. IT-366-07) and Ministerio de Ciencia e Inovación (Grant No. FIS201019609-C02-00). V.C. gratefully acknowledges G. Benedek for fruitful discussions.
${ }^{1}$ Y. L. Chen, J. G. Analytis, J.-H. Chu, Z. K. Liu, S.-K. Mo, X. L. Qi, H. J. Zhang, D. H. Lu, X. Dai, Z. Fang, S. C. Zhang, I. R. Fisher, Z. Hussain, and Z.-X. Shen, Science 325, 178 (2009).

${ }^{2}$ Y. Xia, D. Qian, D. Hsieh, L. Wray, A. Pal, H. Lin, A. Bansil, D. Grauer, Y. S. Hor, R. J. Cava, and M. Z. Hasan, Nat. Phys. 5, 398 (2009).

${ }^{3}$ D. Hsieh, Y. Xia, D. Qian, L. Wray, F. Meier, J. H. Dil, J. Osterwalder, L. Patthey, A. V. Fedorov, H. Lin, A. Bansil, D. Grauer, Y. S. Hor, R. J. Cava, and M. Z. Hasan, Phys. Rev. Lett. 103, 146401 (2009).

${ }^{4}$ K. Kuroda, M. Arita, K. Miyamoto, M. Ye, J. Jiang, A. Kimura, E. E. Krasovskii, E. V. Chulkov, H. Iwasawa, T. Okuda, K. Shimada, Y. Ueda, H. Namatame, and M. Taniguchi, Phys. Rev. Lett. 105, 076802 (2010).
${ }^{5}$ R. Caracas and X. Gonze, Phys. Chem. Miner. 32, 295 (2005).

${ }^{6}$ H. Zhang, C.-X. Liu, X.-L. Qi, X. Dai1, Z. Fang, and S.-C. Zhang, Nat. Phys. 4, 438 (2009).

${ }^{7}$ J. Chang, L. F. Register, S. K. Banerjee, and B. Sahu, Phys. Rev. B 83, 235108 (2011).

${ }^{8}$ W. Zhang, R. Yu, H.-J. Zhang, X. Dai, and Z. Fang, New J. Phys. 12, 065013 (2010)

${ }^{9}$ S. V. Eremeev, Y. M. Koroteev, and E. V. Chulkov, JETP Lett. 91, 387 (2010).

${ }^{10}$ S. V. Eremeev et al., Nat. Commun. 3, 635 (2012).

${ }^{11}$ L.-L. Wang and D. D. Johnson, Phys. Rev. B 83, 241309 (2011).

${ }^{12}$ T. V. Menshchikova, S. V. Eremeev, and E. V. Chulkov, JETP Lett. 94, 106 (2011). 
${ }^{13}$ M. Neupane, S.-Y. Xu, L. A. Wray, A. Petersen, R. Shankar, N. Alidoust, C. Liu, A. Fedorov, H. Ji, J. M. Allred, Y. S. Hor, T.-R. Chang, H.-T. Jeng, H. Lin, A. Bansil, R. J. Cava, and M. Z. Hasan, Phys. Rev. B 85, 235406 (2012).

${ }^{14}$ K. Miyamoto, A. Kimura, T. Okuda, H. Miyahara, K. Kuroda, H. Namatame, M. Taniguchi, S. V. Eremeev, T. V. Menshchikova, E. V. Chulkov, K. A. Kokh, and O. E. Tereshchenko, Phys. Rev. Lett. 109, 166802 (2012).

${ }^{15} \mathrm{~T}$. V. Menshchikova (private communication).

${ }^{16}$ G. J. Snyder and E. S. Toberer, Nat. Mater. 7, 105 (2008).

${ }^{17}$ S. K. Mishra, S. Satpathy, and O. Jepsen, J. Phys.: Condens. Matter 9, 461 (1997).

${ }^{18}$ T. Zhang, P. Cheng, X. Chen, J. F. Jia, X. Ma, K. He, L. Wang, H. Zhang, X. Dai, Z. Fang, X. Xie, and Q.-K. Xue, Phys. Rev. Lett. 103, 266803 (2009).

${ }^{19}$ T. Zhang, P. Cheng, X. Chen, J.-F. Jia, X. Ma, K. He, L. Wang, H. Zhang, X. Dai, Z. Fang, X. Xie, and Q.-K. Xue, Nat. Mater. 9, 225 (2009).

${ }^{20}$ S. Y. F. Zhao, C. Beekman, L. J. Sandilands, J. E. J. Bashucky, D. Kwok, N. Lee, A. D. LaForge, S. W. Cheong, and K. S. Burch, Appl. Phys. Lett. 98, 141911 (2011).

${ }^{21}$ D. Teweldebrhan, V. Goyal, and A. A. Balandin, Nano Lett. 10, 1209 (2010).

${ }^{22}$ W. Dang, H. Peng, H. Li, and Z. Liu, Nano Lett. 10, 2870 (2010).

${ }^{23}$ J. Zhang, Z. Peng, A. Soni, Y. Zhao, Y. Xiong, J. Wang, M. S. Dresselhaus, and Q. Xiong, Nano Lett. 11, 2407 (2011).

${ }^{24}$ K. M. F. Shahil, M. Z. Hossain, D. Teweldebrhan, and A. A. Balandin, Appl. Phys. Lett. 96, 153103 (2010).

${ }^{25}$ L. M. Goncalves, P. Alpuim, A. G. Rolo, and J. H. Correia, Thin Solid Films 519, 4152 (2011).

${ }^{26}$ H. Rauh, R. Geick, H. Köhler, N. Nücker, and N. Lehner, J. Phys. C 14, 2705 (1981).

${ }^{27}$ X. Zhu, L. Santos, R. Sankar, S. Chikara, C. Howard, F. C. Chou, C. Chamon, and M. El-Batanouny, Phys. Rev. Lett. 107, 186102 (2011).

${ }^{28}$ W. Cheng and S.-F. Ren, Phys. Rev. B 83, 094301 (2011).

${ }^{29}$ B.-T. Wang and P. Zhang, Appl. Phys. Lett. 100, 082109 (2012).

${ }^{30}$ B. Qiu and X. Ruan, Phys. Rev. B 80, 165203 (2009).

${ }^{31}$ G. C. Sosso, S. Caravati, and M. Bernasconi, J. Phys.: Condens. Matter 21, 095410 (2009).

${ }^{32}$ K. A. Kokh, B. G. Nenashev, A. E. Kokh, and G. Y. Shvedenkov, J. Cryst. Growth 275, E1964 (2005).
${ }^{33}$ P. Giannozzi et al., J. Phys.: Condens. Matter 21, 395502 (2009).

${ }^{34}$ A. M. Rappe, K. M. Rabe, E. Kaxiras, and J. D. Joannopoulos, Phys. Rev. B 41, 1227 (1990).

${ }^{35}$ A. M. Rappe, K. M. Rabe, E. Kaxiras, and J. D. Joannopoulos, Phys. Rev. B 44, 13175 (1991).

${ }^{36}$ J. P. Perdew and A. Zunger, Phys. Rev. B 23, 5048 (1981).

${ }^{37}$ J. P. Perdew, K. Burke, and M. Ernzerhof, Phys. Rev. Lett. 77, 3865 (1996).

${ }^{38}$ R. W. G. Wyckoff, Crystal Structures, Vol. 2 (Wiley and Sons, New York, 1964).

${ }^{39}$ Y. Kim, X. Chen, Z. Wang, J. Shi, I. Miotkowski, Y. P. Chen, P. A. Sharma, A. L. L. Sharma, M. A. Hekmaty, Z. Jiang, and D. Smirnov, Appl. Phys. Lett. 100, 071907 (2012).

${ }^{40}$ Y. Liang, W. Wang, B. Zeng, G. Zhang, Y. Song, X. Zhang, J. Huang, J. Li, and T. Li, Solid State Commun. 151, 704707 (2011).

${ }^{41}$ W. Richter, H. Köhler, and C. R. Becker, Phys. Status Solidi B 84, 619 (1977).

${ }^{42}$ W. Kullman, J. Geurts, W. Richter, N. Lehner, H. Rauh, U. Steinberger, G. Eichhorn, and R. Geick, Phys. Solidi B $\mathbf{1 2 5}$, 131 (1984).

${ }^{43}$ R. Heid, K.-P. Bohnen, I. Y. Sklyadneva, and E. V. Chulkov, Phys. Rev. B 81, 174527 (2010).

${ }^{44}$ L. E. Diaz-Sánchez, A. H. Romero, and X. Gonze, Phys. Rev. B 76, 104302 (2007).

${ }^{45}$ I. Y. Sklyadneva, R. Heid, K.-P. Bohnen, V. Chis, V. A. Volodin, K. A. Kokh, O. E. Tereshchenko, P. M. Echenique, and E. V. Chulkov, Phys. Rev. B 86, 094302 (2012).

${ }^{46}$ V. Chis, B. Hellsing, G. Benedek, M. Bernasconi, E. V. Chulkov, and J. P. Toennies, Phys. Rev. Lett. 101, 206102 (2008).

${ }^{47}$ V. Chis, B. Hellsing, G. Benedek, M. Bernasconi, E. V. Chulkov, and J. P. Toennies, Phys. Rev. Lett. 103, 069902(E) (2009).

${ }^{48}$ C. S. Jayanthi, H. Bilz, W. Kress, and G. Benedek, Phys. Rev. Lett. 59, 795 (1987)

${ }^{49}$ I. Y. Sklyadneva, G. Benedek, E. V. Chulkov, P. M. Echenique, R. Heid, K.-P. Bohnen, and J. P. Toennies, Phys. Rev. Lett. 107, 095502 (2011).

${ }^{50}$ G. Benedek, M. Bernasconi, V. Chis, E. V. Chulkov, P. M. Echenique, B. Hellsing, and J. P. Toennies, J. Phys.: Condens. Matter 22, 084020 (2010).

${ }^{51}$ D. Campi, M. Bernasconi, and G. Benedek, Phys. Rev. B 86, 075446 (2012).

${ }^{52}$ V. Chis, G. Benedek, P. M. Echenique, and E. V. Chulkov [Phys. Rev. B (to be published)]. 